\title{
Beyond the uterine environment: a nonhuman primate model to investigate maternal-fetal and neonatal outcomes following chronic intrauterine infection
}

\author{
Meredith A. Kelleher ${ }^{1}$, Zheng Liu ${ }^{2}$, Xiaojie Wang ${ }^{2}$, Christopher D. Kroenke², Lisa A. Houser ${ }^{3}$, Brandy L. Dozier ${ }^{3}$, \\ Lauren D. Martin ${ }^{3}$, Ken B. Waites ${ }^{4}$, Cindy McEvoy ${ }^{5}$, Robert L. Schelonka ${ }^{5}$ and Peta L. Grigsby ${ }^{1,6}$
}

BACKGROUND: Intrauterine infection is a significant cause of early preterm birth. We have developed a fetal-neonatal model in the rhesus macaque to determine the impact of chronic intrauterine infection with Ureaplasma parvum on early neonatal reflexes and brain development.

METHODS: Time-mated, pregnant rhesus macaques were randomized to be inoculated with U. parvum (serovar 1; $10^{5}$ c.f.u.) or control media at $\sim 120$ days' gestational age (dGA). Neonates were delivered by elective hysterotomy at 135-147 dGA (term =167d), stabilized, and cared for in our nonhuman primate neonatal intensive care unit. Neonatal reflex behaviors were assessed from birth, and fetal and postnatal brain magnetic resonance imaging (MRI) was performed.

RESULTS: A total of 13 preterm and 5 term macaque infants were included in the study. Ten preterm infants survived to 6 months of age. U. parvum-infected preterm neonates required more intensive respiratory support than did control infants. MRI studies suggested a potential perturbation of brain growth and white matter maturation with exposure to intra-amniotic infection.

CONCLUSION: We have demonstrated the feasibility of longitudinal fetal-neonatal studies in the preterm rhesus macaque after chronic intrauterine infection. Future studies will examine long-term neurobehavioral outcomes, cognitive development, neuropathology, and in vivo brain imaging to determine the safety of antenatal antibiotic treatment for intrauterine infection.

$I^{n}$ ntrauterine infection can lead to premature labor and preterm birth during early gestation (1). Ureaplasma spp. are microbes most commonly associated with preterm birth. They have been detected in $47 \%$ of placentae from women in preterm labor with histological chorioamnionitis (2). Early preterm birth ( $<28$ weeks' gestation) is associated with high mortality and morbidity in survivors as well as with a disproportionate share of perinatal health-care costs (3). Bronchopulmonary dysplasia and neurodevelopmental abnormalities are the sequelae of early prematurity associated with intrauterine infectious and inflammatory processes (4).

Studies from our laboratory have established a nonhuman primate (NHP) model of infection-induced preterm birth using intra-amniotic inoculation with U. parvum (5). This maternal-fetal model results in a robust intrauterine proinflammatory response, a sequential upregulation of amniotic fluid (AF) leukocytes, pro-inflammatory cytokines, prostaglandin E2 and F2 $\alpha$, and matrix metalloproteinase-9, and increased uterine activity leading to premature labor (5). Histological chorioamnionitis and a systemic fetal inflammatory response, fetal lung injury, and pneumonitis were also observed. Clinically, isolation of Ureaplasma spp. in AF from preterm births is associated with adverse psychomotor development and increased risk for cerebral palsy diagnosis at 2 years of age (6). In addition, intra-amniotic $U$. parvum inoculation has been associated with central microgliosis and disrupted neuronal development in the neocortex of fetal mouse brains (7). However, the long-term, neurodevelopmental effects of perinatal Ureaplasma infections have not been adequately studied (8).

This paper describes our new model for the assessment of postnatal outcomes following chronic intra-amniotic infection with $U$. parvum. This model enables the longitudinal study of physiological and neurodevelopmental consequences of infection-associated preterm birth and provides a platform for the assessment of insults across the perinatal period, along with evaluation of new antenatal and postnatal therapeutic strategies.

\section{METHODS}

Maternal-Fetal Animal Model of Chronic Intra-Amniotic Infection

Study protocols were approved by the Oregon National Primate Research Center Institutional Animal Care and Use Committee.

\footnotetext{
'Division of Reproductive and Developmental Sciences, Oregon National Primate Research Center, Beaverton, Oregon; ${ }^{2}$ Advanced Imaging Research Center and Division of Neuroscience, Oregon National Primate Research Center, Beaverton, Oregon; ${ }^{3}$ Division of Comparative Medicine, Oregon National Primate Research Center, Beaverton, Oregon; ${ }^{4}$ Department of Pathology, University of Alabama at Birmingham School of Medicine, Birmingham, Alabama; ${ }^{5}$ Department of Pediatrics, Division of Neonatology, Oregon Health and Science University, Portland, Oregon; ${ }^{6}$ Department of Obstetrics and Gynecology, Oregon Health and Science University, Portland, Oregon. Correspondence: Meredith A. Kelleher (kellehem@ohsu.edu) 
Strict guidelines for humane care were followed, with adherence to the Public Health Service Policy on the Humane Care and Use of Laboratory Animals. Animals were allocated to the study by assignment from the Oregon National Primate Research Center breeding colony. Time-mated pregnant rhesus monkeys (Macaca mulatta) were adapted to a vest and mobile catheter protection device (9), and intrauterine surgery was performed at a mean of 106 dGA (range: day $101-111 ; n=12$ ) to implant catheters in the amniotic cavity and in the maternal femoral vein and artery. Pre- and post-operative Cefazolin sodium (Apotex, Weston, FL) was administered intravenously to prevent infection, and tocolytic medications (e.g., Terbutaline sulfate and/or Atosiban) were used to control postsurgical uterine irritability as previously published $(5,10)$. The animals were divided into three groups as shown in Figure 1, with experiments performed concurrently.

Microorganism. As previously published $(5,10)$, a low-passaged clinical isolate of U. parvum (serovar 1; Diagnostic Mycoplasma Laboratory, University of Alabama, Birmingham, AL) was utilized. To induce chronic U. parvum IAI, intra-amniotic inoculations with $1.4 \times 10^{5} \mathrm{CFU} / \mathrm{ml}$ were performed at $124 \pm 1.9$ days' gestation. Sterile media were administered to gestation-age-matched controls.

\section{Maternal-Fetal Physiological Monitoring and Sampling}

Uterine activity. Intra-amniotic pressure was recorded, digitized, and analyzed using the PowerLab system and LabChartPro (ADInstruments, Colorado Springs, CO) (10). The integrated area under the curve for intra-amniotic pressure was used as the measure of uterine activity and reported as the mean of the cumulative HCA $(\mathrm{mm} \mathrm{Hg} \cdot \mathrm{s} / \mathrm{h})$. The average peak HCA per day prior to inoculation (with U. parvum or media control) represents baseline contractility and was compared with the peak HCA per day from inoculation until delivery (post inoculation).

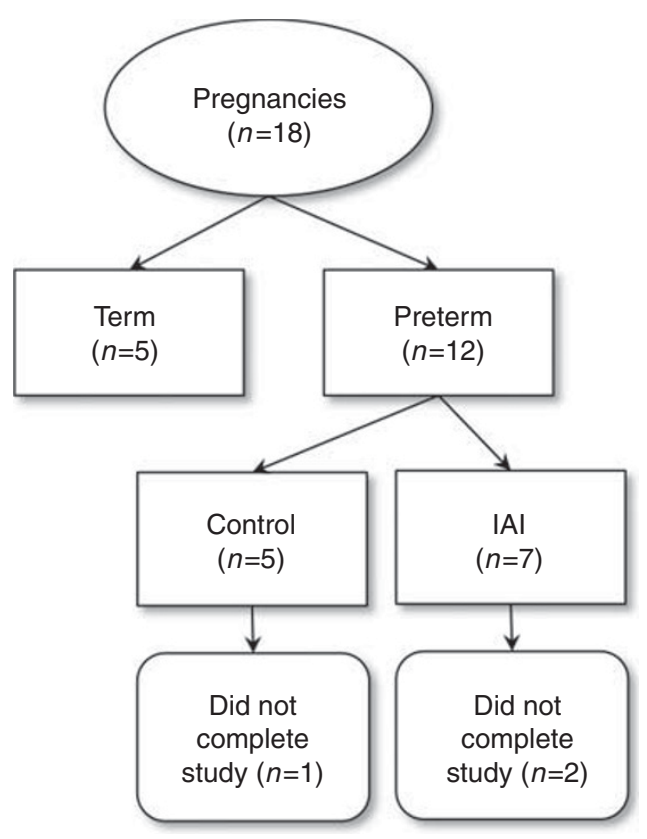

Figure 1. Flowchart of the study groups. Preterm infants from catheterized pregnancies were randomized to control and intra-amniotic infection (IAI). An additional group of term control animals were obtained from non-catheterized pregnancies from the Oregon National Primate Research Center (ONPRC) colony.
AF cytokines. AF was sampled at $0,12,24,48$, and $72 \mathrm{~h}$ post inoculation and then twice weekly. AF concentrations of the pro-inflammatory mediators IL-1 $\beta$, IL- 6 , tumor necrosis factor- $\alpha$, and prostaglandins $\mathrm{PGE}_{2}$ and $\mathrm{PGF}_{2 \alpha}$ were determined using commercially available human or rhesus monkey-specific enzymelinked immunosorbent assay kits (Life Technologies, Grand Island, NY) previously validated for use in the rhesus monkey $(5,10,11)$. The minimum detectable levels of IL-1 $\beta$, IL-6, and tumor necrosis factor$\alpha$ assays were $<1-2 \mathrm{pg} / \mathrm{ml}$. The inter-assay coefficient of variance was below $15 \%$ for all assays.

Culture and PCR detection of U. parvum. Quantitative cultures/PCR for $U$. parvum were performed on samples of $\mathrm{AF}$, placenta, fetal membranes, and neonatal nasal swabs $(10,12,13)$.

\section{Preterm Delivery and Neonatal Intensive Care}

Elective hysterotomies were performed for delivery of preterm infants between 135 and $147 \mathrm{dGA}(n=12)$. All preterm neonates were immediately transferred to our Special Care Nursery for resuscitation and stabilization. Control and IAI preterm infants received prophylactic postnatal azithromycin $(20 \mathrm{mg} / \mathrm{kg} / \mathrm{day}$, intravenous for 5 days, Fresenius Kabi USA, Grand Island, NY) to reflect standard clinical practice for suspected neonatal Ureaplasma infection and to prevent secondary pneumonitis $(14,15)$.

Respiratory stabilization and support. A standardized neonatal resuscitation algorithm (modified Neonatal Resuscitation Program (16)) was utilized to ensure consistency across the experimental groups. Neonatal resuscitation began with room air (21\% inspired oxygen concentration $\left.\left(\mathrm{FiO}_{2}\right)\right)$ and positive pressure ventilation using a Neo-Tee infant T-piece Resuscitator (Mercury Medical, Clearwater, FL). Targeted peak inspiratory pressure $\left(20-30 \mathrm{~cm} / \mathrm{H}_{2} \mathrm{O}\right)$ and positive end-expiratory pressure $\left(5 \mathrm{~cm} / \mathrm{H}_{2} \mathrm{O}\right)$ were maintained. Neonates with clinical evidence of respiratory failure and/or signs of increasing respiratory distress (e.g., tachypnea $>60$ breaths per minute with intercostal retractions, and with required $\mathrm{FiO}_{2}>40 \%$ ) were intubated with a 1.0-2.0 ID endotracheal tube and administered bovine-derived surfactant (100 mg phospholipids/kg of birth weight, $4 \mathrm{ml} / \mathrm{kg}$ intratracheal; Survanta Beractant, AbbVie, North Chicago, IL) and synchronized intermittent mandatory ventilation (40 breaths/min, peak inspiratory pressure $15 \mathrm{~cm} / \mathrm{H}_{2} \mathrm{O}$ and positive end expiratory pressure $5 \mathrm{~cm} / \mathrm{H}_{2} \mathrm{O}$ Servo300 Maquet, Solino, Sweden). Infants were weaned from ventilator support when breathing in excess of the ventilator and oxygen saturation was stable while receiving $\mathrm{FiO}_{2}$ of 0.21 . Newborn Health Assessments (modified APGAR scores (17)) were performed immediately at 5 and 20 min after birth, with a score of 10-12 indicating good physical condition.

Intravenous access and parenteral nutrition. Intravenous access was achieved by placement of a peripheral IV catheter in the distal saphenous or cephalic vein (Introcan Safety IV Catheter, $22 \mathrm{G} \times 1$ ", Teflon Straight, Braun Medical, Bethlehem, PA). Within the first hour of age, a peripherally inserted central catheter (PICC) was also placed in the saphenous vein (First PICC S/L 26GA, 1.9 F, Argon Medical Devices, Athens, TX) for administration of total parenteral nutrition. Radiographs confirmed correct placement and terminus of the PICC within the inferior vena cava at the level of the liver. Standard preterm neonatal total parenteral nutrition multivitamins and trace elements (ExactaMix, Baxter Healthcare Corporation, Englewood, CO) were administered at $120 \mathrm{ml} / \mathrm{kg} /$ day, increasing daily by $20 \mathrm{ml} / \mathrm{kg} /$ day. Enteral feeding with donated human breast milk $(0.5-1.0 \mathrm{ml})$ was introduced every $2 \mathrm{~h}$, over a $3-5$ day period orally or via nasal/oral gastric gavage (3.5 PVC Feeding Tube w/ Radiopaque Line, Covidein, Mansfield, MA) until a suckling reflex was evident. Bovine milk infant formula (Similac for Supplementation, Abbott Nutrition, Lake Forest, IL) was then introduced ad lib, and total intake was adjusted based on parenteral and enteral volumes. Adequate enteral feeding volumes and presence of regular 
bowel movements were criteria for removal of the PICC line and cessation of parenteral feeding.

\section{Infant Behavioral Assessment Scale}

From the day of birth and then every 2-3 days as clinical condition allowed, all infants were assessed using neuromotor, sensory-motor, and basic survival reflex tests (as previously published (17-19)) by trained personnel with interoperator reliability of $>90 \%$. Reflexes tested included rooting (elicited by lightly brushing the cheek), snouting (lightly brushing the nose), suckling (nipple placed in mouth), startle (auditory startle to sudden loud noise), grasping (with each hand or foot), clasping (ability for infant to support bodyweight by holding on to "mother"), proprioception (placing of hands and feet when moving the infant down toward a counter surface), visual orientation and following (near and far distances), and auditory orientation (to lip-smacking with the infant facing away from the tester). The post-conception age at which animals achieved a criterion response for each reflex (using a scoring scale outlined by Sackett et al. (18)) was recorded.

\section{Fetal and Neonatal Brain MRI}

MRI studies were performed on a Siemens 3T Tim Trio system (Erlangen, Germany). In utero examinations of the fetal brain were carried out at $139 \mathrm{dGA}$ following published procedures (20). Anatomical and diffusion MRI data were acquired and analyzed for neonatal brains at term-equivalent age, term plus 1 month, and term plus 6 months of age (Supplementary Methods online).

\section{Statistical Analyses}

Data in the text are presented as mean \pm SD. MRI results are presented for control $(n=3)$, IAI $(n=4)$, and term control animals $(n=3)$. Uterine activity data, colony counts, and pro-inflammatory mediators are shown for control $(n=3)$ and IAI $(n=5)$ animals. Neonatal reflex data are shown for control $(n=4)$, IAI $(n=5)$, and term control $(n=3)$ animals. A single control pregnancy was not catheterized because of previous surgical history of hysterotomy. This animal has been included in postnatal assessments only. Oneway ANOVA, paired $t$-test or two-way ANOVA with repeated measures, and post hoc Sidak's multiple comparison test were used for analyses of neonatal reflexes and AF pro-inflammatory mediators. $P<0.05$ was considered statistically significant. Analyses were performed using Prism v6.0 for MacOSX (GraphPad Software, La Jolla, CA).

\section{RESULTS}

\section{Maternal-Fetal Model of Chronic Intra-Amniotic Infection}

After inoculation with $U$. parvum, there was an exponential growth of microorganisms in the $\mathrm{AF}$ that peaked between $8.4 \times 10^{4}$ and $1.2 \times 10^{6} \mathrm{CFU} / \mathrm{ml}$ of $\mathrm{AF}$ within 4 days and stabilized thereafter (Figure 2). A single animal had peak AF colony growth of $1.1 \times 10^{7} \mathrm{CFU} / \mathrm{ml}$ at 15 days. Interestingly, despite a lower inoculum than used previously (10), colony growth rates and peak levels were comparable to those found in our previous studies. AF, infant nasal/throat swabs, placenta, and fetal membranes were $100 \%$ positive for U. parvum by culture/PCR at the time of delivery. All control animals were negative on culture/PCR. For the neonates that completed the study, the inoculation to delivery interval was $18 \pm 2$ days (range: 15-21 days). The two IAI neonates that were killed before 6 months of postnatal age showed early signs of preterm labor and were delivered after 9 and 13 days of infection, which was attributed to secondary AF infection (Staphylococcus epidermidis) and significant growth restriction, respectively.

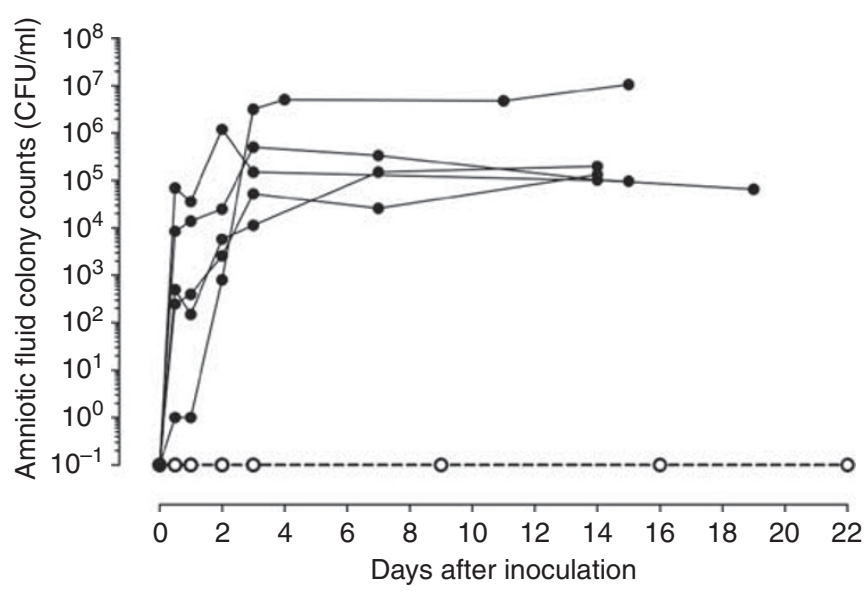

Figure 2. Amniotic fluid Ureaplasma parvum ((CFU/ml) growth curve (log scale)). Data are presented as mean \pm SD for animals following intra-amniotic inoculation (IAl; filled circles) and in gestation-agematched controls (open circles) from inoculation to delivery. All control animals were negative for $U$. parvum for the duration of the experiment.

Before intra-amniotic inoculation, the uterus was quiescent. The maximal hourly contraction area (HCA) per day was calculated for the pre-inoculation baseline and postinoculation periods and are shown in Table 1. The peak daily uterine contractility was significantly elevated in the post-inoculation period for the U. parvum-infected group when compared with baseline. Uterine activity of control animals remained stable with no increase in the period following control inoculation with sterile media. Uterine activity was not determined in uncatheterized term pregnancies.

\section{AF Pro-Inflammatory Mediators}

Coinciding with the increased uterine activity following intraamniotic inoculation with U. parvum, AF concentrations of pro-inflammatory cytokines (tumor necrosis factor- $\alpha$, interleukin (IL)-1 $\beta$, and IL-6) and prostaglandins ( $\mathrm{PGE}_{2}$ and $\mathrm{PGF}_{2 \alpha}$ ) were significantly elevated post inoculation when compared with baseline (both control and IAI) and with media control post-inoculation values (Table $1 ; P<0.05$ ). There were no differences between baseline and postinoculation pro-inflammatory mediators for control animals.

\section{Delivery and Neonatal Outcomes}

The average gestational age at C-section delivery was $144 \pm 2.2$ days for control $(n=5)$ and $141 \pm 4.6$ days in IAI $(n=7)$ animals (term $=\sim 167$ days' gestational age (dGA) (ref. 21). Birth weight was slightly higher in the control group when compared with that in the IAI group (394 \pm 91 and $355 \pm 52 \mathrm{~g}$, respectively); however, this difference was not significant $(P=0.46)$. Of the preterm infants, one control and two IAI animals were euthanized prior to 6 months of age and prior to completion of the study. The control animal that did not complete the study was killed at 9-day postnatal age after developing clinical signs of necrotizing enterocolitis that did 
Table 1. Amniotic fluid pro-inflammatory mediators and uterine activity after intra-amniotic Ureaplasma parvum inoculation

\begin{tabular}{|c|c|c|c|c|}
\hline \multirow[t]{2}{*}{ Variable } & \multicolumn{2}{|c|}{ Control } & \multicolumn{2}{|c|}{ Intra-amniotic infection } \\
\hline & Baseline & Post-inoculation & Baseline & Post-inoculation \\
\hline $\mathrm{PGE}_{2}(\mathrm{pg} / \mathrm{ml})$ & $32 \pm 8$ & $55 \pm 14$ & $80 \pm 23$ & $2,580 \pm 1,045^{a}$ \\
\hline $\mathrm{PGF}_{2 \mathrm{a}}(\mathrm{pg} / \mathrm{ml})$ & $35 \pm 8$ & $84 \pm 22$ & $40 \pm 6$ & $733 \pm 162^{a}$ \\
\hline TNF-alpha (pg/ml) & Not detectable & Not detectable & Not detectable & $1,035 \pm 320^{a}$ \\
\hline IL-1B (pg/ml) & Not detectable & Not detectable & Not detectable & $239 \pm 71^{a}$ \\
\hline IL-6 (ng/ml) & $0.5 \pm 0.1$ & $1.0 \pm 0.7$ & $0.7 \pm 0.1$ & $57.8 \pm 9.3^{\mathrm{a}}$ \\
\hline UA (mm-Hg.s/h) & $1,174 \pm 300$ & $1,256 \pm 285$ & $909 \pm 232$ & $1,545 \pm 260^{b}$ \\
\hline
\end{tabular}

AF, amniotic fluid; CFU, HCA, hourly cumulative area; IA, intra-amniotic infection; IAI, intra-amniotic infection; IL, interleukin; PG, prostaglandin; TNF, tumor necrosis factor; UA, uterine activity.

Data are mean \pm SEM Concentrations of amniotic fluid pro-inflammatory mediators represent the average concentration prior to intra-amniotic inoculation (baseline) and the maximal concentration reached post inoculation. UA is expressed as the average of the maximal HCA under the contraction curve per day prior to inoculation (baseline) and post inoculation. IA animals received inoculation with U. parvum (serovar1) $10^{5} \mathrm{CFU} / \mathrm{ml}$ and gestation-age-matched controls received vehicle inoculation only.

${ }^{a}$ AF pro-inflammatory mediator post-inoculation values were significantly increased from control baseline, control peak, and IAI baseline values

bPost-inoculation UA was significantly increased compared with baseline in the IAI group ( $P<0.05$ paired two-way ANOVA, Šídák's multiple comparison).
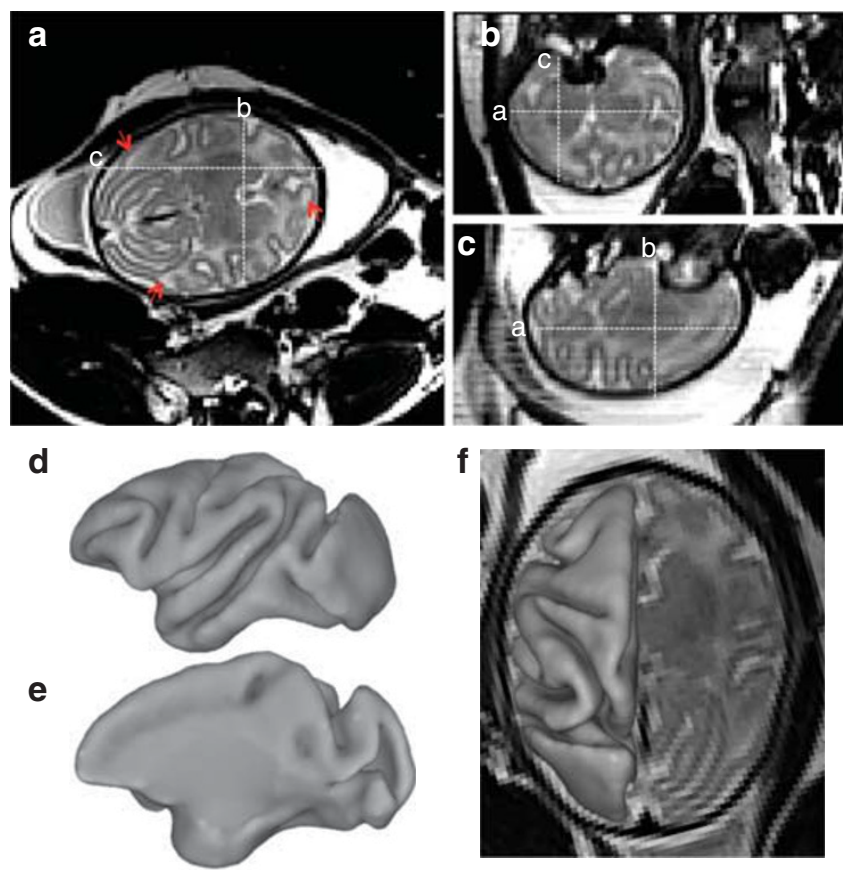

Figure 3. In utero T2-weighted control fetal brain images and cortical surface at 139 days' gestational age (dGA). T2-weighted image was acquired using HASTE along the axial direction. From axial (a), coronal (b), and sagittal (c) views, tissue contrast between cortical gray matter, fetal white matter, and deep nuclei is evident (a-c). Heterogenous signal intensity within the developing cerebral cortex is present at this developmental stage (a, arrowheads). The cerebral cortical surface was generated from segmentations of the T2-weighted image (d-f). At this developmental stage, all primary and secondary sulci and gyri present in adult cortex are identifiable.

not respond to antibiotic treatment $(7$ days of $5 \mathrm{mg} / \mathrm{kg}$ per day S.Q. Gentamicin (Fresenius Kabi, Lake Zurich, IL) and $100 \mathrm{mg} / \mathrm{kg}$ B.I.D. I.V. Ampicillin (Sandoz, Holzkirchen, Germany)) and complete gastrointestinal rest. A second animal from the IAI group that developed necrotizing enterocolitis responded to treatment and completed the study. Two neonatal deaths that occurred in the IAI group at 12 and $21 \mathrm{~h}$ post birth were due to respiratory failure, complicated by secondary infection and growth restriction. Both animals had histologic evidence of atelectasis and pneumonitis present at necropsy.

All infants required resuscitation at delivery and were supported with supplementary oxygen $\left(\mathrm{FiO}_{2}\right.$ up to $\left.0.30-0.40\right)$ and/or mechanical ventilation as indicated by clinical signs. During the first $30 \mathrm{~min}$ of resuscitation and stabilization, $U$. parvum IAI infants required higher concentrations of inspired oxygen compared with preterm control animals to achieve $\mathrm{SpO}_{2}$ levels between 60 and $80 \%\left(\mathrm{FiO}_{2}, 0.49\right.$ vs. 0.37, respectively, $P<0.05)$. Neonatal respiratory distress syndrome, characterized by significant tachypnea, sternal retractions, and labored breathing, was present in three of seven (43\%) IAI infants and one of four (25\%) control preterm infants. The length of ventilation was $2.2 \mathrm{~h}$ for the control infant and an average of $10 \mathrm{~h}$ for all IAI infants or $5.6 \mathrm{~h}$ for only those IAI infants who completed the study. Infants exposed to chronic $U$. parvum IAI required a significantly longer duration of total parenteral nutrition compared with control preterm infants before achieving full enteral feeding $(10.2 \pm 1$ days IAI vs. $4.3 \pm 2$ days control, $P<0.05)$.

\section{Fetal Brain Development}

Figure 3 shows a T2-weighted brain image acquired in utero of a control fetus at 139-dGA. Brain lesions secondary to inflammatory processes can be identified with T2-weighted magnetic resonance imaging (MRI) (22). However, normal variation in white matter (WM) signal intensity associated with brain development can confound lesion identification. High signal intensity is observable in late-developing WM structures such as within the frontal and temporal lobes (Figure 3a, arrowheads). Conspicuous lesions attributable to brain inflammation have not been observed on MRI in the IAI fetuses examined to date. Histological examinations are currently ongoing. As shown in Figure $\mathbf{3 d}-\mathbf{f}$, the threedimensional (3D) T2-weighted images facilitate construction 


\section{Articles | Kelleher et al.}

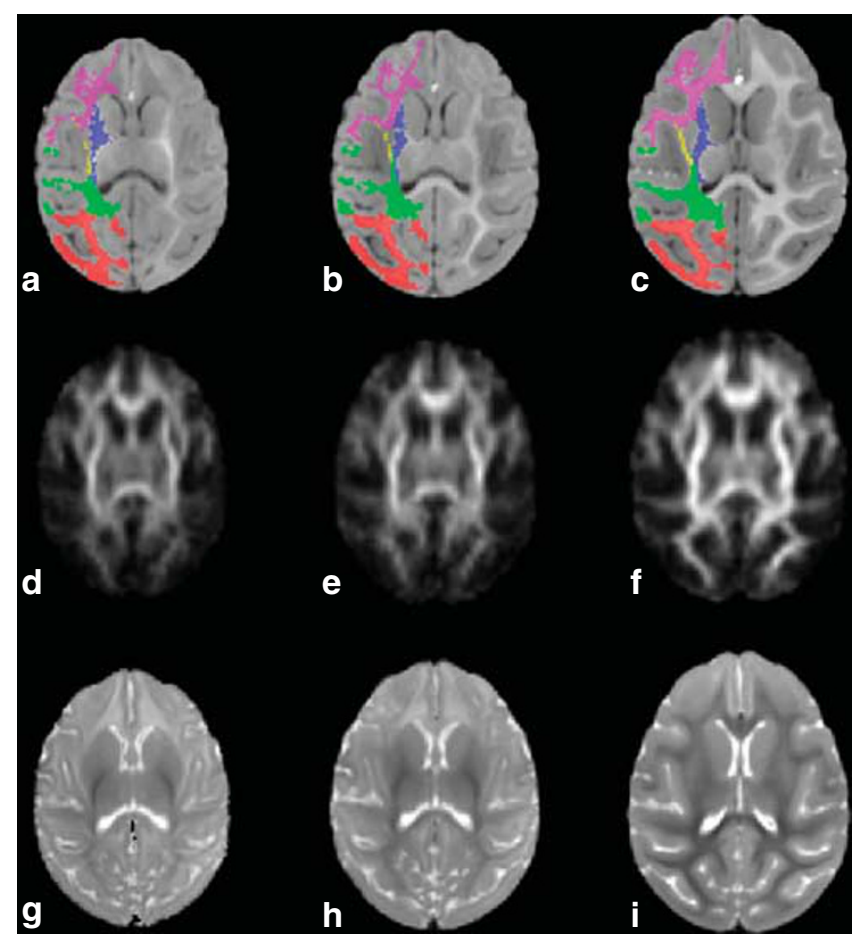

Figure 4. Age-specific reference T1-weighted (a-c), fractional anisotropy $(\mathbf{d}-\mathbf{f})$, and T2-weighted ( $\mathbf{g}-\mathbf{i})$ brain templates were constructed from term control subject scans at term $(\mathbf{a}, \mathbf{d}, \mathbf{g})$, term plus 1 month $(\mathbf{b}, \mathbf{e}, \mathbf{f})$, and term plus 6 months of age $(\mathbf{c}, \mathbf{f}, \mathbf{i})$. For T1-weighted images, six white matter parcellations are overlaid on the left brain hemisphere, where the occipital white matter is in red, parietal white matter in green, internal capsule in blue, external capsule in yellow, temporal white matter in cyan, and frontal white matter in magenta. The T2-weighted images corresponding to a echo time (TE) of $100 \mathrm{~ms}$ were constructed from parameter maps of proton density and T2 determined from the dual echo measurement, as described in Supplementary Methods.

of cerebral cortical surface models, which enable characterization of fetal brain development $(23,24)$.

\section{Neonatal Brain Growth and Development}

Figure 4 shows template T1-weighted (top row), fractional anisotropy (middle row), and T2-weighted (bottom row) image axial views at the three postnatal ages chosen for MRI examination. The six WM subregions are overlaid onto one hemisphere of the T1-weighted images. In addition to overall changes in brain size with development, MRI signal intensity changed within WM for each of the three modalities over the first 6 months of life (Figure 5d).

Brain growth data for term control, control, and IAI groups are shown in Figure 5. Overall brain volume increased with age (data not shown) but decreased with age when normalized to body weight. The brain volume to body weight ratios (corrected to term, term plus 1 month, and term plus 6 months ages) were similar across all groups (Figure 5a), with no significant differences between infants born at term or preterm. As expected, brain volume at 6 months of age was proportional to fetal brain weight measured at necropsy at the same age (Figure $5 \mathbf{b}$ ). Brain volume fractions of gray matter,
WM, hippocampus, and lateral ventricles are shown in Figure $\mathbf{5 c - f}$, and were highly similar between groups. The gray matter volume fraction decreased in all groups from term to 1 month of age and remained stable from 1 to 6 months of age. From term age to 6 months, the volume occupied by WM increased in all groups. No significant differences were observed between IAI and control groups in the volume fraction of the hippocampus and lateral ventricles.

Neuroimaging indices of WM maturation are shown for the internal capsule (Figure 6a,c) and frontal WM (Figure 6b,d). As expected, the water T2 decreased with age (Figure 6a,b), and fractional anisotropy increased with age (Figure $6 c, d$ ) over the first 6 months of life. Within both regions displayed, the average value for the water T2 was slightly longer in IAI animals compared with controls at term-equivalent age, and the average value for fractional anisotropy was slightly lower in IAI animals compared with controls at 1 month of age. These findings suggest perturbed WM maturation in IAI animals relative to controls; however, neither of these differences reached statistical significance.

\section{Infant Behavioral Assessments}

Infant Behavioral Assessment Scale testing was performed on preterm and term neonates with most "survival" and motor reflexes present within the first two postnatal weeks of life. This is consistent with data from Sackett et al. (18) in nurseryreared rhesus infants born between 151 and 161 dGA from a study of assisted reproduction technology. When comparing post-conception age between preterm and term infants in our study (Figure 7), term control animals reached criterion at significantly later post-conception age (but similar postnatal age) for startle, rooting, suckling, righting, grasping, clasping, and auditory orient reflexes when compared with both preterm groups $(P<0.05)$. Criterion for grasp reflex developed significantly earlier in the post-conception age in IAI animals compared with controls $(P<0.001)$, potentially due to earlier gestational age at delivery in this group as postnatal ages at criterion were similar. Unlike survival and motor reflexes that assess maturity of spinal and subcortical nervous system functions, the placing reflex and sensorimotor visual reflexes that require more complex neural mechanisms developed at a similar post-conception age for both term and preterm cohorts despite differences in gestational age at birth.

\section{DISCUSSION}

This study outlines the successful expansion of our maternalfetal model of intra-amniotic $U$. parvum infection to allow postnatal assessments of NHP infant outcomes following preterm birth. Neonatal NHP studies, including those of respiratory therapies and brain development in the preterm baboon and behavioral development in pigtail macaques, have previously demonstrated the value of NHP models for the study of important perinatal outcomes that are difficult to assess clinically (18,25-29). By combining our established catheterized pregnant monkey model (10) with our newly 


\section{NHP model of infection and prematurity $\mid$ Articles}
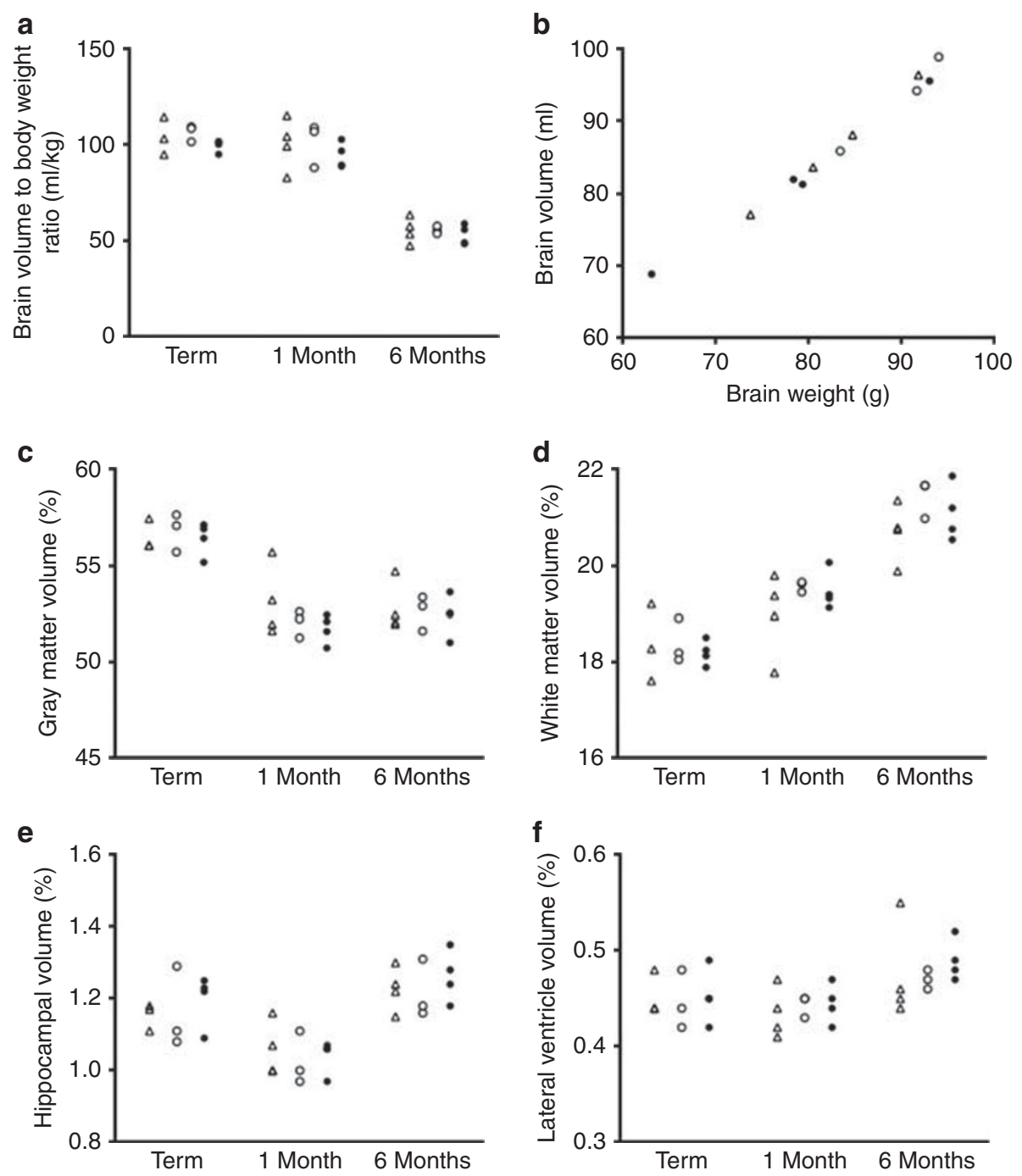

Figure 5. Comparison of average age-related total and regional brain volumes for control and intra-amniotic infection (IAI) groups. (a) The brain volumes at term, term plus 1 month, and term plus 6 months of age are shown for term control (triangle), control (open circle), and intra-amniotic infection (IAl; filled circle). (b) Brain volumes calculated from magnetic resonance imaging (MRI) is linearly related with brain weight at the same age measured at necropsy. Regional brain volumes as a proportion of total brain volume are shown for gray matter (c), white matter (d), hippocampus (e), and lateral ventricle (f).

developed NHP Special Care Nursery, we are able to examine longitudinal outcomes in preterm rhesus monkeys across the fetal and postnatal periods. The long-term goal of these studies is to assess more complex aspects of postnatal cognitive development following infection-associated preterm birth and the safety of potential antenatal antibiotic treatments.

This study demonstrates our ability to perform serial in vivo fetal and postnatal MRI studies of the developing rhesus brain. Fetal MRI is a valuable diagnostic tool for predicting future neurobehavioral outcomes in preterm infants with reduced white and gray matter volumes indicative of fetal brain injury and insults such as periventricular leukomalacia $(30,31)$. Although our studies have not identified significant differences on MRI, minor changes in brain volume and development warrant further investigation. By combining clinically relevant MR imaging modalities and advances in technology and analysis techniques with an experimental NHP model of intrauterine infection, we are able to leverage the power of a controlled experimental setting with data that are highly translatable into clinical practice.

We also provide early neonatal reflex data for infant monkeys following infection-associated preterm birth. In the current study, development of reflexes requiring simpler neural processing, such as "survival" reflexes, were associated with postnatal age in term and preterm infants. These reflexes were present soon after birth despite differences in gestational age. However, more complex reflexes requiring integration of sensory and motor functions across a number of brain regions, such as visual following and placing (18), developed at similar post-conception ages in both term and preterm control or IAI infants. This suggests that premature birth 


\section{Articles | Kelleher et al.}
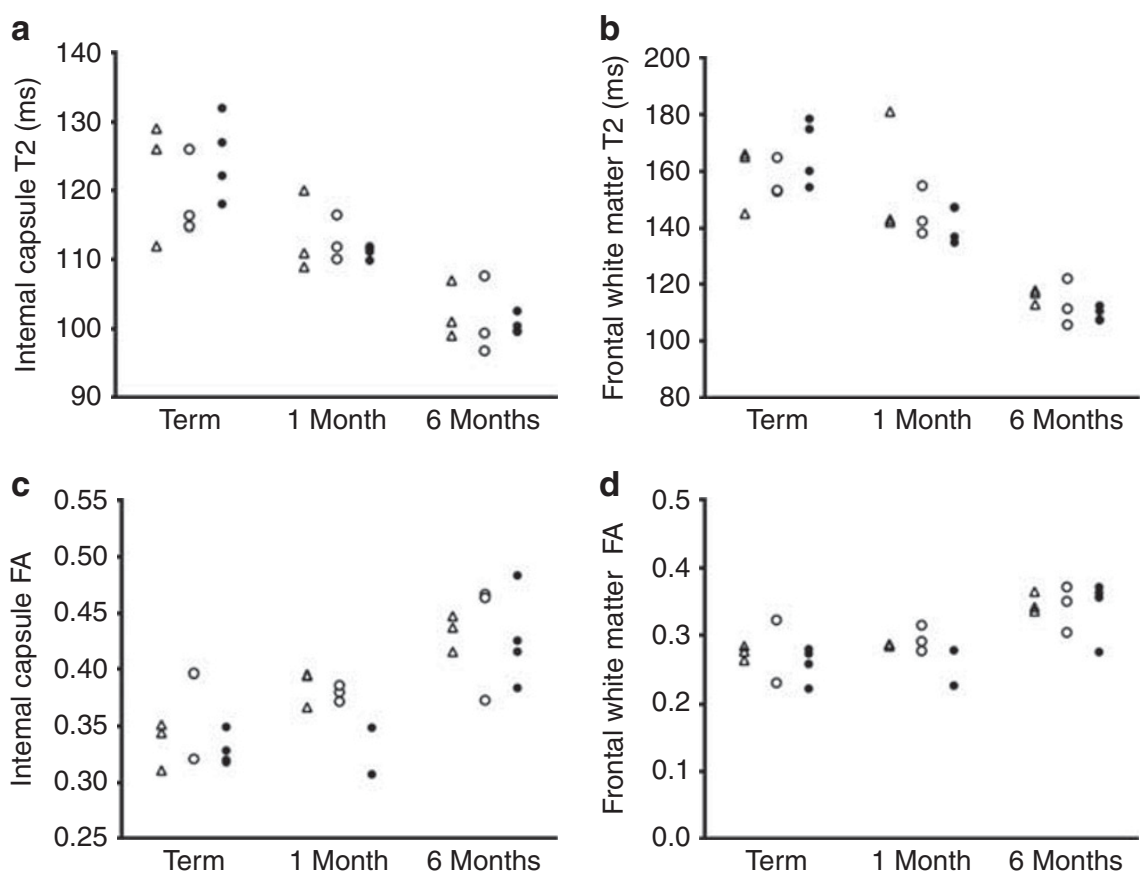

Figure 6. Comparison of age-related average regional T2 and fractional anisotropy (FA) values between control and intra-amniotic infection (IAI) groups. T2 and FA values in the internal capsule $(\mathbf{a}, \mathbf{c})$ and the frontal white matter (b,d) regions are shown at term, term plus 1 month, and term plus 6 months of age for term control (triangle), control (open circle), and IAl (filled circle) animals.

and/or infection in these monkeys did not delay the development of these later neonatal reflexes. Small differences in gestational age and bodyweight at birth between animals in the preterm control and IAI groups are a potential source of variation between the groups. However, no associations between birth weight and the acquisition of neonatal reflexes were identified (data not shown). In addition, the necessary use of postnatal antibiotics to treat Ureaplasma-infected infants (and therefore also preterm controls) and clinical use for treatment of necrotizing enterocolitis may be considered a potential confounder, but represents normal clinical outcomes and practice. Although we have not identified marked differences in early reflex outcomes, ongoing examination of recognition and procedural memory, which require more advanced prefrontal cortical, striatal, and cerebellar maturity, may identify functional differences in preterm infants not observed in the early neonatal period.

The current study characterizes a chronic intra-amniotic infection resulting in long-term exposure of the fetus to infection and inflammation. We previously inoculated rhesus monkeys with $10^{7}$ or $10^{5} \mathrm{CFU} / \mathrm{ml}$ U. parvum at 128 or 138 dGA, respectively $(5,10)$. Despite differences in inoculum size, AF colony counts reached similar peak levels around $10^{6} \mathrm{CFU} / \mathrm{ml}$ across studies $(5,10)$. Ammonia produced by Ureaplasma spp. has been shown to reach inhibitory concentrations in in vitro culture media $(5,32)$ and likely inhibits growth in AF. However, we also observed a muted response in AF pro-inflammatory mediator concentrations in the chronic model compared with short-term infection.
Gestational age may mediate this effect, with the inoculation-to-delivery interval and production of proinflammatory mediators decreasing as age at inoculation increases $(5,10)$. This is consistent with increased sensitivity of the uterus to inflammatory (pro-labor) mediators and heightened uterine contractility as gestation progresses. Chronic exposure to inflammation, beginning at an earlier gestational age, likely mimics clinical cases of intrauterine infection, with microorganisms detected in AF as early as 16 weeks' gestation in women, many weeks before the onset of labor (33). The length of in utero exposure to U. parvum and inflammation may also mediate the severity of fetal brain injury and level of postnatal functional deficits associated with intrauterine inflammation (34-37). The mechanisms of perinatal inflammatory brain injury remain poorly understood. Comorbidities including prematurity, hypoxicischemic insults, intrauterine growth restriction, neonatal resuscitation efforts, and polymicrobial infection further complicate our understanding of these processes, particularly with respect to the "second-hit hypothesis", in which perinatal inflammation may increase vulnerability to injury from other fetal or postnatal sources.

These studies have focused on the need to characterize the neurodevelopmental consequences of intrauterine infection. However, the authors recognize the importance of perinatal infection in the etiology of preterm lung injury and bronchopulmonary dysplasia (38). A technical limitation of this study is that volume-targeted ventilation was not used. Our use of respiratory support was determined by the clinical 

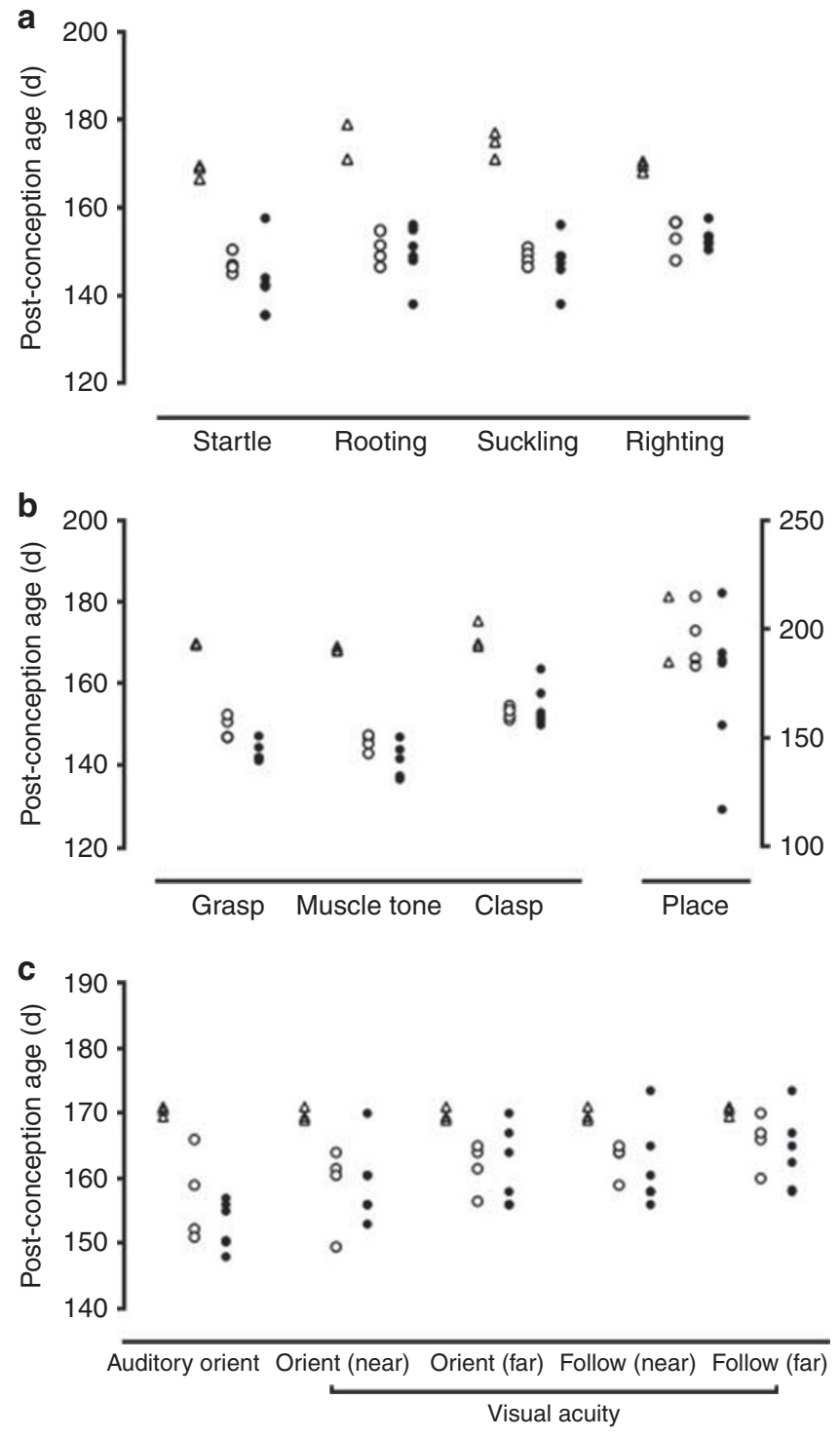

Figure 7. Neonatal reflex behaviors. Post-conception age at which criterion was reached for (a) survival, (b) motor (values for placing are plotted against the right $y$-axis), and (c) sensorimotor neonatal reflexes for term control (triangles), control (open circles), and intra-amniotic infection (IAl; filled circles) animals. Term control animals reached criterion at significantly later post-conception age (but similar postnatal age) for startle, rooting, suckling, righting, grasping, clasping, and auditory orient reflexes when compared with preterm control and IAI animals. The criterion for grasp reflex developed significantly earlier in IAI animals compared with that in controls. $P<0.05$, ANOVA.

needs of each infant based on Neonatal Resuscitation Guidelines (16). Steps were taken to reduce the $\mathrm{FiO}_{2}$ and duration of intubation and invasive respiratory support. Continuous mask continuous positive airway pressure was not required beyond the resuscitation period, but continuous positive airway pressure was used intermittently $(5 \mathrm{~min}$ intervals) in the first $48 \mathrm{~h}$ of life in response to apneic events or precipitous drop in $\mathrm{SpO}_{2}$. Although lung inflammation and pathology have not been reported in this study, future work will include assessments of lung injury and function in these animals, including consideration of the greater level of respiratory support required by IAI infants and the consequences this may have on both lung and brain development $(26,39,40)$.

We have previously shown in a fetal rhesus study that intraamniotic $U$. parvum infection leads to a robust lung inflammatory response and that maternal azithromycin treatment can ameliorate this injury and also eradicate $U$. parvum from the $\mathrm{AF}$ and fetal tissues, resulting in a prolongation of gestation (10). However, the importance of providing clear evidence of functional outcomes has been highlighted by the Oracle Trials, due to the potential effect antenatal antibiotic exposure may have on neurodevelopmental outcomes $(41,42)$. Correlating functional and histological findings is a priority of future studies utilizing this new NHP model in order to examine the long-term safety of antimicrobial interventions for premature birth.

The development of this fetal-neonatal model demonstrates our ability to study the intra-uterine environment, during infection and pharmaceutical or physiological interventions, providing direct physiological measurements across gestation. By combining these measurements with assessments of postnatal functional outcomes correlated with both in vivo imaging techniques and in vitro assays, we have the ability to assess a broad range of insults across the perinatal period. The emergence of new threats such as the Zika virus highlights the need for basic research utilizing relevant animal models with the ability to advance our understanding and treatment of human obstetrical and perinatal questions.

\section{SUPPLEMENTARY MATERIAL}

Supplementary material is linked to the online version of the paper at http://www.nature.com/pr

\section{ACKNOWLEDGMENTS}

We acknowledge the contributions of Kimberly Ray, Edward Henson, Norma Drinkwater, Nikki Sternberger, Sheila Roberts, Megan O'Brien, and the Clinical Medicine, Surgical Services and Behavioral Services Units, Division of Comparative Medicine, Oregon National Primate Research Center, L. Xiao, Ms Donna Crabb, and Ms Amy Ratliff (University of Alabama at Birmingham) and Tom Burbacher (Director of the Infant Primate Research Laboratory, WaNPRC, Seattle).

Portions of this work have been presented at the 61st annual meeting of the Society for Reproductive Investigation, Florence, Italy, March 2014; at the European Workshop on Neonatal Transition, Prato, Italy, August 2014; and at the 41st annual meeting of the Fetal and Neonatal Physiological Society, St Vincent, Italy, September 2014.

\section{STATEMENT OF FINANCIAL SUPPORT}

This work was supported by the following grants: Collins Medical Trust (pilot program funding), National Institute of Child Health and Human Development HD055053, and the National Institutes of Health, number 8P51 OD 011092-53.

Disclosure: The authors declare no conflict of interest.

\section{REFERENCES}

1. Goldenberg RL, Culhane JF, Iams JD, Romero R. Epidemiology and causes of preterm birth. Lancet 2008;371:75-84. 
2. Hillier SL, Martius J, Krohn M, Kiviat N, Holmes KK, Eschenbach DA. A case-control study of chorioamnionic infection and histologic chorioamnionitis in prematurity. N Engl J Med 1988;319:972-8.

3. Saigal S, Doyle LW. An overview of mortality and sequelae of preterm birth from infancy to adulthood. Lancet 2008;371:261-9.

4. Dammann O, Leviton A, Gappa M, Dammann CE. Lung and brain damage in preterm newborns, and their association with gestational age, prematurity subgroup, infection/inflammation and long term outcome. BJOG 2005;112 (Suppl 1): 4-9.

5. Novy MJ, Duffy L, Axthelm MK, et al. Ureaplasma parvum or Mycoplasma hominis as sole pathogens cause chorioamnionitis, preterm delivery, and fetal pneumonia in rhesus macaques. Reprod Sci 2009;16:56-70.

6. Berger A, Witt A, Haiden N, et al. Intrauterine infection with Ureaplasma species is associated with adverse neuromotor outcome at 1 and 2 years adjusted age in preterm infants. J Perinat Med 2009;37:72-8.

7. Normann E, Lacaze-Masmonteil T, Eaton F, Schwendimann L, Gressens P, Thebaud B. A novel mouse model of Ureaplasma-induced perinatal inflammation: effects on lung and brain injury. Pediatr Res 2009;65:430-6.

8. Viscardi RM. Ureaplasma species: role in neonatal morbidities and outcomes. Arch Dis Child Fetal Neonatal Ed 2014;99:F87-92.

9. Gravett MG, Witkin SS, Haluska GJ, Edwards JL, Cook MJ, Novy MJ. An experimental model for intraamniotic infection and preterm labor in rhesus monkeys. Am J Obstet Gynecol 1994;171:1660-7.

10. Grigsby PL, Novy MJ, Sadowsky DW, et al. Maternal azithromycin therapy for Ureaplasma intraamniotic infection delays preterm delivery and reduces fetal lung injury in a primate model. Am J Obstet Gynecol 2012;207:e1-14.

11. Sadowsky DW, Novy MJ, Witkin SS, Gravett MG. Dexamethasone or interleukin-10 blocks interleukin-1beta-induced uterine contractions in pregnant rhesus monkeys. Am J Obstet Gynecol 2003;188:252-63.

12. Waites KB, Duffy LB, Talkington DF, Schwartz SB. Mycoplasma pneumoniae, Mycoplasma hominis and Ureaplasma Cultures from Clinical Specimens. In: Garcia LS, Isenberg HD (eds). Clinical Microbiology Procedures Handbook. 3rd (edn). ASM Press: United States of America, 2010, pp 3.15.1-3.15.15.

13. Xiao L, Glass JI, Paralanov V, et al. Detection and characterization of human Ureaplasma species and serovars by real-time PCR. J Clin Microbiol 2010;48:2715-3.

14. Viscardi RM, Othman AA, Hassan HE, et al. Azithromycin to prevent bronchopulmonary dysplasia in Ureaplasma-infected preterm infants: pharmacokinetics, safety, microbial response, and clinical outcomes with a 20-milligram-per-kilogram single intravenous dose. Antimicrob Agents Chemother 2013;57:2127-33.

15. Hassan HE, Othman AA, Eddington ND, et al. Pharmacokinetics, safety, and biologic effects of azithromycin in extremely preterm infants at risk for Ureaplasma colonization and bronchopulmonary dysplasia. J Clin Pharmacol 2011;51:1264-75.

16. Textbook of Neonatal Resuscitation, 6th edn. American Academy of Pediatrics and American Heart Association: 2011.

17. Ruppenthal GC, Sackett GP. Research Protocol And Technician's Manual, 2nd edn. Seattle, Washington: Infant Primate Research Laboratory, University of Washington, 1992.

18. Sackett G, Ruppenthal G, Hewitson L, Simerly C, Schatten G. Neonatal behavior and infant cognitive development in rhesus macaques produced by assisted reproductive technologies. Dev Psychobiol 2006;48:243-65.

19. Schneider ML, Coe CL, Lubach GR. Endocrine activation mimics the adverse effects of prenatal stress on the neuromotor development of the infant primate. Dev Psychobiol 1992;25:427-39.

20. Wang X, Pettersson DR, Studholme C, Kroenke CD. Characterization of laminar zones in the mid-gestation primate brain with magnetic resonance imaging and histological methods. Front Neuroanat 2015;9:147.

21. Novy MJ, Walsh SW. Dexamethasone and estradiol treatment in pregnant rhesus macaques: effects on gestational length, maternal plasma hormones, and fetal growth. Am J Obstet Gynecol 1983;145:920-31.
22. Dean JM, van de Looij Y, Sizonenko SV, et al. Delayed cortical impairment following lipopolysaccharide exposure in preterm fetal sheep. Ann Neurol 2011;70:846-56.

23. Dubois J, Benders M, Borradori-Tolsa C, et al. Primary cortical folding in the human newborn: an early marker of later functional development. Brain 2008;131:2028-41.

24. Lefevre J, Germanaud D, Dubois J, et al. Are developmental trajectories of cortical folding comparable between cross-sectional datasets of fetuses and preterm newborns? Cereb Cortex 2016;26:3023-5.

25. Yoder BA, Coalson JJ. Animal models of bronchopulmonary dysplasia. The preterm baboon models. Am J Physiol Lung Cell Mol Physiol 2014;307:L970-7.

26. Verney C, Rees S, Biran V, Thompson M, Inder T, Gressens P. Neuronal damage in the preterm baboon: impact of the mode of ventilatory support. J Neuropathol Exp Neurol 2010;69:473-82.

27. Kroenke CD, Van Essen DC, Inder TE, Rees S, Bretthorst GL, Neil JJ. Microstructural changes of the baboon cerebral cortex during gestational development reflected in magnetic resonance imaging diffusion anisotropy. J Neurosci 2007;27:12506-5.

28. Meyer J, Grant K, Burbacher T, et al. Relationship between prenatal cortisol exposure and behavioral development in macaque monkeys. Psychoneuroendocrinology 2015;61:30-1.

29. Golub MS, Hogrefe CE, Vandevoort CA. Binge drinking prior to pregnancy detection in a nonhuman primate: behavioral evaluation of offspring. Alcohol Clin Exp Res 2014;38:551-6.

30. Peterson BS, Anderson AW, Ehrenkranz R, et al. Regional brain volumes and their later neurodevelopmental correlates in term and preterm infants. Pediatrics 2003;111:939-48.

31. Inder TE, Huppi PS, Warfield S, et al. Periventricular white matter injury in the premature infant is followed by reduced cerebral cortical gray matter volume at term. Ann Neurol 1999;46:755-60.

32. Cassell GH, Blanchard A, Duffy L. Clinical and Pathogenic Microbiology. Mosby-Year Book: St Louis, CA, 1994.

33. Cassell GH, Davis RO, Waites KB, et al. Isolation of Mycoplasma hominis and Ureaplasma urealyticum from amniotic fluid at 16-20 weeks of gestation: potential effect on outcome of pregnancy. Sex Transm Dis 1983;10:294-302.

34. Malaeb S, Dammann O. Fetal inflammatory response and brain injury in the preterm newborn. J Child Neurol 2009;24:1119-26.

35. Kuban KC, O'Shea TM, Allred EN, et al. The breadth and type of systemic inflammation and the risk of adverse neurological outcomes in extremely low gestation newborns. Pediatr Neurol 2015;52:42-8.

36. Dada T, Rosenzweig JM, Al Shammary M, et al. Mouse model of intrauterine inflammation: sex-specific differences in long-term neurologic and immune sequelae. Brain Behav Immun 2014;38:142-50.

37. Dammann O, Leviton A. Intermittent or sustained systemic inflammation and the preterm brain. Pediatr Res 2014;75:376-80.

38. Viscardi RM, Kallapur SG. Role of Ureaplasma respiratory tract colonization in bronchopulmonary dysplasia pathogenesis: current concepts and update. Clin Perinatol 2015;42:719-38.

39. Rees SM, Loeliger MM, Munro KM, et al. Cerebellar development in a baboon model of preterm delivery: impact of specific ventilatory regimes. J Neuropathol Exp Neurol 2009;68:605-15.

40. Loeliger M, Inder T, Cain S, et al. Cerebral outcomes in a preterm baboon model of early versus delayed nasal continuous positive airway pressure. Pediatrics 2006;118:1640-53.

41. Kenyon S, Pike K, Jones DR, et al. Childhood outcomes after prescription of antibiotics to pregnant women with spontaneous preterm labour: 7year follow-up of the ORACLE II trial. Lancet 2008;372:1319-27.

42. Kenyon S, Pike K, Jones DR, et al. Childhood outcomes after prescription of antibiotics to pregnant women with preterm rupture of the membranes: 7-year follow-up of the ORACLE I trial. Lancet 2008;372: 1310-8. 